\title{
Direct Treatment Costs of Invasive Candidiasis in Haematology Patients at a South African Private Hospital
}

\author{
Rozlyn Cruickshank $^{1} \&$ Fatima Suleman ${ }^{1}$ \\ ${ }^{1}$ Discipline of Pharmaceutical Sciences, School of Health Sciences, University of KwaZulu-Natal, South Africa \\ Correspondence: Professor Fatima Suleman, Discipline of Pharmaceutical Sciences, University of KwaZulu-Natal, \\ Private Bag X54001, Durban, South Africa, 4000. Tel: 27-31-260-7941. E-mail: sulemanf@ukzn.ac.za
}

Received: January 8, 2019 Accepted: May 20, 2019 Online Published: June 10, 2019

doi:10.5539/gjhs.v11n7p60 URL: https://doi.org/10.5539/gjhs.v11n7p60

\begin{abstract}
Background: Haematology patients are at a high risk of developing invasive candidiasis (IC). Fluconazole has been the mainstay of prophylaxis and treatment with a newer class of therapeutic options, the echinocandins, having seen a considerable improvement in treatment success. However, these agents are associated with substantial acquisition costs when compared to fluconazole.
\end{abstract}

Objective: This study analysed the direct treatment costs of invasive candidiasis in haematology patients, comparing the costs between three groups depending on the treatment, namely, the fluconazole only group, the echinocandin only group or the group that was treated with both fluconazole and an echinocandin. To determine which variables contributed to the overall costs and whether there were differences between the groups.

Methods: This was a retrospective, single-centre economic analysis at a private hospital of patients with IC in the haematology ward in Durban, KwaZulu-Natal Province, South Africa. The direct medical costs related to managing IC were analysed. Adult patients ( $\geq 18$ years old) diagnosed with a haematology disorder and a positive blood culture for Candida who were prescribed fluconazole and/or an echinocandin as treatment were included in the study. Patients treated with echinocandins, fluconazole or both classes of antifungals were analysed separately and compared.

Results: No statistically significant difference for duration of antifungal treatment or length of hospital stay between the three groups existed. Mean overall direct treatment costs per patient were: ZAR130 326 (95\% CI: -4 932 - 265 584) for patients treated with fluconazole, ZAR241 165 (95\% CI: 159175 - 323 155) for patients receiving an echinocandin and ZAR270 802 (95\% CI: 68277 - 473 327) for patients treated with the combination

Conclusion: The results of this cost analysis found that treatment with fluconazole only is considerably less expensive, almost half of the mean daily treatment cost, than an echinocandin only and treatment using both agents, is less expensive than an echinocandin only as first-line therapy.

Keywords: cost, echinocandin, fluconazole, haematology, invasive candidiasis

\section{Introduction}

Candidaemia is the fourth most common hospital acquired bloodstream infection in the United States of America (USA) and the most frequently occurring worldwide, with its frequency rising rapidly (Kontoyiannis, 2001). According to the Lancet laboratories Annual Surveillance Report and Antibiotic Guide (2017), Candida species are also the fourth most frequently isolated organism from blood cultures in the private sector in KwaZulu-Natal Province, South Africa. Invasive candidiasis (IC) is an important clinical entity, specifically among critically ill Intensive Care Unit (ICU) patients, with crude mortality rates of 40-60\% (Playford, Lipman, \& Sorrell, 2010). Invasive fungal infection poses a serious risk to critically ill and immunocompromised patients, particularly hematopoietic stem cell transplant (HSCT) recipients and those who have received intensive chemotherapy for acute leukaemia (Cornely et al., 2011). These patients experience febrile neutropenia, which complicates the differential diagnosis between a fungal infection and colonisation (Gedik et al., 2014). The outcome of IC is dependent on early initiation of effective antifungal therapy as inadequate first line treatment results in a significant increase in mortality (Glöckner \& Karthaus, 2011). The main therapeutic agents that are currently used to treat IC include broad-spectrum oral and parenteral triazoles such as fluconazole and itraconazole, lipid formulations of amphotericin B, and the newest class of antifungals, echinocandins, including caspofungin, 
micafungin and anidulafungin (Kontoyiannis, 2001). The growing incidence of fungal infections, and the expenditure related to their treatment, have been increasing worldwide (Gedik et al., 2014). IC, along with life-threatening complications, are associated with increased hospital length of stay, costly care in the ICU and the use of expensive antifungal agents, resulting in a significant rise in healthcare costs (Heimann et al., 2014). The objectives of this study was to conduct a cost analysis of the direct treatment costs of invasive candidiasis in haematology patients, to assess whether the choice of treatment had an impact on the length of hospital stay and to determine which of the direct costs contributed the most to the overall cost of treating an episode of IC. This study utilizes the perspective of a South African private hospital, and included direct medical costs, specifically hospital ward fees, medication acquisition and administration, haematology consultation and laboratory blood culture costs. This assessment is important in order to understand the contributing cost drivers in an episode of IC, and to use the data to establish improved and more cost-effective treatment strategies in high risk haematology patients.

\section{Materials and Methods}

\subsection{Study Design}

This was a descriptive, retrospective, non-interventional, cost analysis study using quantitative data from patient files to compare the direct treatment cost of treating IC in high-risk haematology patients. These patients were defined as having an existing haematology condition, such as lymphoma, leukemia and myeloma, which results in them being immune compromised and thus increases their likelihood of invasive fungal infections. The patients were divided into three groups according to the antifungal medicine they were prescribed during their admission: fluconazole, echinocandin or both medicines.

\subsection{Study Setting and Population}

The study took place in a private urban hospital in Durban, South Africa, which consists of 36 beds, of which 12 are used exclusively for haematology patients. Data was collected from the $1^{\text {st }}$ August 2015 to $31^{\text {st }}$ August 2017, with all patients admitted during this period, and meeting the inclusion criteria, being included in the study. The following inclusion criteria applied, adult patients (aged 18 years and older) with an existing haematology diagnosis, such as lymphoma, leukaemia, myeloma, haemolytic anaemia and those undergoing HSCT, with a positive blood culture for Candida. The following exclusion criteria applied, patients who died before being treated with an antifungal agent and those who were treated with an antifungal other than an echinocandin and/or fluconazole.

\subsection{Data Collection}

Data was extracted from the electronic and paper medical records for all patients meeting the inclusion criteria that were maintained on the private institution's information system, specifically those from $1^{\text {st }}$ August 2015 to $31^{\text {st }}$ August 2017. A data collection sheet was created as the data extraction instrument, with the following variables being extracted: Demographic data (age, gender and underlying haematology diagnosis, name of medical aid); hospital data (admission and discharge date, total length of stay, length of stay in each level of care (i.e. general, high care, isolation and ICU), number of blood cultures during hospital admission, and number of doses of fluconazole and an echinocandin that were administered).

\subsection{Costing}

Only direct medical costs were considered for this study, the South African Rand (ZAR) to United States Dollar (USD) average exchange was ZAR13.83 in 2015, ZAR14.71 in 2016 and ZAR13.21 in 2017. Included in the direct medical costs were the antifungal medications and consumables used for their administration (which are detailed below), blood cultures, haematology consultations and hospitalisation costs. Direct non-medical costs and indirect medical costs, as well as intangible costs, were not included in the study. The antifungal administration costs were based on the Single Exit Price (SEP) of the antifungal medication, as obtained from the Medicine Price database for the years 2015, 2016 and 2017. Single Exit Price refers to section 22G of the Medicine Amendment Act which came into effect on the $2^{\text {nd }}$ May 2004 mandating that medicine manufacturers may only sell their products at one price to all customers. The term means that each product and its variants 'exits' the factory at one single price for that product or variant. The SEP is adjusted annually taking into consideration the different criteria as laid out in regulations. The daily administration consumable costs included the cost of a medication administration pack, specific for each antifungal, which included the agreed costs charged to the medical fund for the syringes, needles, alcohol swabs, diluents, infusion fluids and infusion sets required for the daily administration of the antifungal medication. The total daily cost (Table 1), included the sum of the SEP of the antifungal agent and the administration consumable costs multiplied by the prescribed daily dosage.

Table 1. Antifungal medication and consumable costs (ZAR) equivalent to USD 2015 $=13.83 ; 2016=14.71 ; 2017$ 
$=13.21$

\begin{tabular}{|c|c|c|c|c|c|c|c|c|c|c|}
\hline \multirow{4}{*}{ Fluconazole } & \multirow{4}{*}{ (Dilfucan®) } & \multicolumn{9}{|c|}{ Antifungal costs } \\
\hline & & \multicolumn{3}{|l|}{2015} & \multicolumn{3}{|l|}{2016} & \multicolumn{3}{|l|}{2017} \\
\hline & & $\begin{array}{l}\text { SEP } \\
\text { (Single } \\
\text { Exit } \\
\text { Price) }\end{array}$ & $\begin{array}{l}\text { Daily } \\
\text { administration } \\
\text { consumable } \\
\text { costs }\end{array}$ & $\begin{array}{l}\text { Total } \\
\text { daily } \\
\text { cost }\end{array}$ & $\begin{array}{l}\text { SEP } \\
\text { (Single } \\
\text { Exit } \\
\text { Price) }\end{array}$ & $\begin{array}{l}\text { Daily } \\
\text { administration } \\
\text { consumable } \\
\text { costs }\end{array}$ & $\begin{array}{l}\text { Total } \\
\text { daily } \\
\text { cost }\end{array}$ & $\begin{array}{l}\text { SEP } \\
\text { (Single } \\
\text { Exit } \\
\text { Price) }\end{array}$ & $\begin{array}{l}\text { Daily } \\
\text { administration } \\
\text { consumable } \\
\text { costs }\end{array}$ & $\begin{array}{l}\text { Total } \\
\text { daily } \\
\text { cost }\end{array}$ \\
\hline & & 197.29 & 368.52 & 1131.61 & 206.74 & 737.03 & 1150.51 & 212.72 & 737.03 & 1162.47 \\
\hline \multirow{4}{*}{ Echinocandin } & $\begin{array}{l}\text { Caspofungin } \\
\left.\text { (Cancidas }{ }^{\circledR}\right)\end{array}$ & 2888.60 & 396.12 & 3284.72 & & & & & & \\
\hline & $\begin{array}{l}\text { Anidulafungin } \\
\left(\text { Eraxis }{ }^{\circledR}\right)- \\
\text { loading dose } \\
(\text { day } 1)\end{array}$ & 4838.96 & 390.80 & 5229.76 & & & & & & \\
\hline & $\begin{array}{l}\text { Anidulafungin } \\
\left.\text { (Eraxis }{ }^{\circledR}\right) \\
\text { maintenance } \\
\text { dose }\end{array}$ & 2419.48 & 387.58 & 2807.06 & & & & & & \\
\hline & $\begin{array}{l}\text { Micafungin } \\
\left(\text { Mycamine }{ }^{\circledR}\right)\end{array}$ & 1824.00 & 435.94 & 2259.94 & 1824.00 & 436.72 & 2260.72 & 1960.80 & 438.34 & 2399.14 \\
\hline
\end{tabular}

Table 2. Cost of hospital stay and laboratory costs (ZAR) equivalent to USD $2015=13.83 ; 2016=14.71 ; 2017=$ 13.21

\begin{tabular}{llllll}
\hline \multicolumn{2}{l}{ Hospital rates* } & & & \\
\hline Date & General & Isolation & High Care $(\mathrm{HC})$ & ICU & Laboratory rates \\
\hline 2015 & 1932.40 & 2545.85 & 5129.80 & 8614.65 & 212.25 \\
2016 & 2224.40 & 2887.14 & 5903.92 & 9785.70 & 220.85 \\
2017 & 2329.95 & 3002.45 & 6066.65 & 10362.48 & 229.75 \\
\hline
\end{tabular}

*Average costs were calculated for each level of care for a particular year based on the individual medical aid tariffs applicable to each patient included in the study.

Table 3. Haematologist inpatient consultation costs (ZAR) equivalent to USD $2015=13.83 ; 2016=14.71 ; 2017=$ 13.21

\begin{tabular}{|c|c|c|c|c|c|c|c|c|}
\hline \multicolumn{9}{|c|}{ Haematologist inpatient consultation rates* } \\
\hline & \multicolumn{4}{|l|}{ Initial } & \multicolumn{4}{|c|}{ Follow up } \\
\hline & General & Isolation & $\mathrm{HC}$ & ICU & General & Isolation & $\mathrm{HC}$ & ICU \\
\hline 2015 & 619.05 & 619.05 & 391.58 & 1305.75 & 263.89 & 263.89 & 391.58 & 652.87 \\
\hline 2016 & 564.13 & 564.13 & 401.29 & 1343.02 & 285.44 & 285.44 & 401.29 & 671.5 \\
\hline 2017 & 656.15 & 656.15 & 458.09 & 1527.17 & 340.21 & 340.21 & 458.09 & 761.85 \\
\hline
\end{tabular}

*Average costs were calculated based on the individual medical aid tariffs applicable to each patient included in the study.

The hospital ward charges were based on the National Health Network agreed tariffs with the individual medical aids for that specific year, the laboratory costs for a blood culture (Table 2) and the haematologist consultation rates (Table 3) were based on the agreed fees by the hospital with the medical funders for each year of the study. 


\subsection{Data Analysis}

The data analysis consisted of two components, the first entailing an analysis of the data to establish any statistically significant relationships between the variables. The second was a sensitivity analysis that increased and decreased each cost parameter to identify the factor that had the greatest impact on the total cost of treating an IC episode.

Statistical analysis was performed using IBM SPSS Statistics software, for Windows version 25. One-way ANOVA was applied to test the statistical significance of the normally distributed continuous variables between the three groups. Dichotomous variables were tested using Chi squared, with a $p$-value of $<0.05$ being considered significant. For descriptive purposes, patient and cost data are presented as the median and range or the mean and $95 \%$ confidence interval (CI), as appropriate.

One-way sensitivity analysis was performed using Microsoft ${ }^{\circledR}$ Excel 2010, by increasing and decreasing each direct cost parameter over a range between $5 \%$ and $20 \%$, while keeping the other costs constant, observing the effect of the results and identifying which variable had the greatest impact on the total cost of treating an episode of IC. The following factors were analysed with the one-way sensitivity analysis:

1) Mean ICU ward costs

2) Mean high care ward costs

3) Mean isolation ward costs

4) Mean general ward costs

5) Mean antifungal medication administration costs for the treatment duration

6) Mean laboratory culture costs

7) Mean haematologist consultation costs

\section{Results}

The final dataset included any patient over the age of 18 years old with an underlying haematology diagnosis and a positive blood culture for Candida. A total of 321 patient admissions were identified that included treatment with fluconazole and/or an echinocandin during the study period from 1 August 2015 to 31 August 2017. Of these, 96 episodes were excluded based on the exclusion criteria regarding age and underlying haematology diagnosis. Of the remaining 225 episodes, only 24 had a positive blood culture for Candida and were therefore eligible to be included in the cost analysis.

\subsection{Demographic Data}

The median age of the patients was 54 years, 44 years and 38.5 years in the fluconazole, echinocandin and both groups respectively. Of the 24 patients, $33.3 \%(n=8)$ were female, and the most common underlying haematology diagnoses were acute myeloid leukaemia $(\mathrm{n}=6)$, acute lymphoblastic leukaemia $(\mathrm{n}=4)$ and multiple myeloma $(\mathrm{n}=$ 4) (Table 4). The one-way ANOVA showed no statistically significant difference in baseline characteristics in terms of median age between the three groups. The Chi-Square test showed no statistically significant difference between the groups with regards to gender and haematology diagnosis. 
Table 4. Patient characteristics and underlying haematology diagnosis

\begin{tabular}{llll}
\hline & \multicolumn{1}{l}{ Fluconazole, $\mathrm{N}=5$} & Echinocandins, $\mathrm{N}=11$ & Both, $\mathrm{N}=8$ \\
\cline { 2 - 4 } & $\mathrm{n}(\%)$ & $\mathrm{n}(\%)$ & $\mathrm{n}(\%)$ \\
\cline { 2 - 4 } & & & \\
\hline Age (years), median (range) & $54(27-84)$ & $44(19-71)$ & $38.5(18-77)$ \\
\hline Female & $3(60)$ & $3(27.3)$ & $2(25)$ \\
\hline Haematology diagnosis & & & $1(12.5)$ \\
\hline Acute promyelocytic leukaemia & - & - & - \\
\hline Acute lymphoblastic leukaemia & $1(20)$ & $3(27.3)$ & $2(25)$ \\
\hline Acute Myeloid Leukaemia & $1(20)$ & $3(27.3)$ & $1(12.5)$ \\
\hline Burkitt Lymphoma & $1(20)$ & - & $2(25)$ \\
\hline Diffuse large B cell lymphoma & - & - & - \\
\hline Haemolytic anaemia & - & $1(9.1)$ & - \\
\hline Hepatosplenic T cell lymphoma & - & $1(9.1)$ & $2(25)$ \\
\hline Hodgkin lymphoma & - & - & - \\
\hline Multiple myeloma & $1(20)$ & $3(27.3)$ & - \\
\hline Non Hodgkin lymphoma & $1(20)$ & - & \\
\hline
\end{tabular}

\#One way ANOVA; ¥Chi-Square.

\subsection{Statistical Analysis}

Patients receiving both fluconazole and an echinocandin had a much longer duration of antifungal treatment as well as overall hospital stay (Table 5). The patients who were treated with fluconazole only had much shorter antifungal treatment duration and an overall hospital stay. The patients receiving an echinocandin had the shortest general ward stay but the longest ICU stay. One way ANOVA showed no statistical significant difference for duration of antifungal treatment or length of hospital stay between the three groups.

Table 5. Duration of antifungal treatment and length of hospital stay

\begin{tabular}{lllll}
\hline & Fluconazole, N=5 & Echinocandins, N=11 & Both, N=8 & $p$ value \\
\hline Duration of antifungal treatment (days), mean & 8.4 & 18 & 22.3 & 0.135 \\
Overall hospital stay (days), mean & 23.3 & 28.45 & 38.69 & 0.197 \\
Length of stay - General ward (days), mean & 10.3 & 4.3 & 10.75 & 0.179 \\
Length of stay - Isolation ward (days), mean & 5 & 5.9 & 6.75 & 0.942 \\
Length of stay - High Care (days), mean & 2.7 & 6.05 & 10.5 & 0.243 \\
Length of stay - ICU (days), mean & 5.3 & 12.23 & 10.69 & 0.659 \\
\hline
\end{tabular}

The mean overall direct treatment costs per patient were, ZAR130 326 (95\% CI: -4 932 - 265 584) for patients treated with fluconazole, ZAR241 165 (95\% CI: 159175 - 323 155) for those receiving an echinocandin and ZAR270 802 (95\% CI: 68277 - 473 327) for patients treated with both (Table 6). There is an excess cost of ZAR110 839 per patient in the echinocandin group and of ZAR140 476 in the group treated with both medications compared to the fluconazole group. The mean direct costs per day were ZAR5 615 (95\% CI: $1000-10229)$ in the fluconazole group vs ZAR8 450 (95\% CI: 6582 - 10 317) in the echinocandin group and ZAR6 717 (95\% CI: $4248-9186$ ) in the group with both medications. Antifungal administration treatment costs contributed $7.5 \%$ in the fluconazole group, $17.4 \%$ in the echinocandin group and $16.5 \%$ in the group treated with both, to the overall direct costs. The mean number of antifungal treatment days in the fluconazole group was 8.4 with a mean daily cost of ZAR1 160 (95\% CI: 1153 - 1166), 28.5 days in the echinocandin group with a mean daily cost of 
ZAR2 365 (95\%CI: 2218 - 2511) and 18 days with a mean daily cost of ZAR2 106 (95\% CI: $1546-2667, \mathrm{P}=<$ $0.001)$ in the combined treatment group.

Table 6. Overview of the direct cost distribution among groups

\begin{tabular}{|c|c|c|c|c|}
\hline \multicolumn{5}{|c|}{ Direct cost parameter $(\mathrm{ZAR})$, mean $(95 \% \mathrm{CI})$} \\
\hline & Fluconazole, $\mathrm{N}=5$ & Echinocandins, $\mathrm{N}=11$ & Both, $\mathrm{N}=8$ & p value* \\
\hline \multicolumn{5}{|l|}{ Ward costs } \\
\hline \multirow{2}{*}{ General } & 23660 & 9537 & 22526 & \multirow{2}{*}{0.201} \\
\hline & $(-9639-56960)$ & $(2179-16896)$ & $(6882-38170)$ & \\
\hline \multirow{2}{*}{ Isolation } & 14539 & 17042 & 17630 & \multirow{2}{*}{0.975} \\
\hline & $(16024-45102)$ & $(-1747-35832)$ & $(925-34335)$ & \\
\hline \multirow{2}{*}{ High care } & 16379 & 35832 & 60092 & \multirow{2}{*}{0.291} \\
\hline & $(-29098-61858)$ & $(20704-68959)$ & $(15250-104935)$ & \\
\hline \multirow{2}{*}{$\mathrm{ICU}$} & 54921 & 119692 & 107756 & \multirow{2}{*}{0.692} \\
\hline & $(-93994-203836)$ & $(43856-195529)$ & $(-43781-259295)$ & \\
\hline \multirow{2}{*}{ Total ward costs } & 109500 & 182105 & 208006 & \multirow{2}{*}{0.488} \\
\hline & $(-10675-229677)$ & $(116573-247638)$ & $(34518-381494)$ & \\
\hline \multirow{2}{*}{ Haematologist consultation costs } & 10259 & 15281 & 15953 & \multirow{2}{*}{0.514} \\
\hline & $(1237-19282)$ & $(10600-19961)$ & $(5769-26136)$ & \\
\hline \multirow{2}{*}{ Antifungal treatment costs } & 9742 & 41886 & 44803 & \multirow{2}{*}{$0.032 *$} \\
\hline & $(3069-16415)$ & $(23404-60367)$ & $(25484-64121)$ & \\
\hline \multirow{2}{*}{ Blood culture costs } & 823 & 1892 & 2039 & \multirow{2}{*}{0.134} \\
\hline & $(340-1306)$ & $(1098-2686)$ & $\left(\begin{array}{llll}1 & 043-3035)\end{array}\right.$ & \\
\hline \multirow{2}{*}{ Total direct costs } & 130326 & 241165 & 270802 & \multirow{2}{*}{0.348} \\
\hline & $(-4932-265584)$ & $(159175-323155)$ & $(68277-473327)$ & \\
\hline \multirow{2}{*}{ Daily direct costs } & 5615 & 8450 & 6717 & \multirow{2}{*}{0.206} \\
\hline & $(1000-10229)$ & $(6582-10317)$ & $(4248-9186)$ & \\
\hline
\end{tabular}

* = statistically significant.

\subsection{Sensitivity Analysis}

One-way sensitivity analysis was performed in Microsoft $₫$ Excel by varying the mean cost of each parameter of the three groups over a range between $5 \%$ and $20 \%$. Changes in the mean total direct costs base value (fluconazole $=$ ZAR130 326, echinocandin = ZAR241 165 and both = ZAR270 802) were noted. The one way sensitivity analysis showed that the mean ICU and high care ward costs were considerable cost drivers across all three groups over the $7.5 \%, 10 \%, 15 \%$ and $20 \%$ changes. This is in line with the data that showed that ward costs contributed the most towards the overall cost of treating an episode of IC. Medicine administration costs for the duration of therapy were an important cost driver in the echinocandin only group and the combined treatment group, but not in the fluconazole group (Table 7). This is due to the much higher acquisition costs of the echinocandins compared to fluconazole. 
Table 7. One way sensitivity results

\begin{tabular}{|c|c|c|c|c|c|c|c|c|c|c|c|c|c|c|c|}
\hline \multirow{4}{*}{ MEAN COSTS } & \multicolumn{15}{|c|}{ \% CHANGE IN TOTAL DIRECT COSTS } \\
\hline & 20 & 15 & 10 & 7.5 & 5 & 20 & 15 & 10 & 7.5 & 5 & 20 & 15 & 10 & 7.5 & 5 \\
\hline & $\%$ & $\%$ & $\%$ & $\%$ & $\%$ & $\%$ & $\%$ & $\%$ & $\%$ & $\%$ & $\%$ & $\%$ & $\%$ & $\%$ & $\%$ \\
\hline & \multicolumn{5}{|c|}{ FLUCONAZOLE } & \multicolumn{6}{|c|}{ ECHINOCANDIN } & \multicolumn{4}{|c|}{ ВОТн } \\
\hline $\mathrm{ICU}$ & 8.4 & 5.3 & 3.5 & 2.6 & 1.8 & 9.9 & 6.2 & 4.1 & 3.1 & 2.1 & 8.0 & 6.0 & 4.0 & 3.0 & 2.0 \\
\hline High Care & 2.5 & 1.6 & 1.0 & 0.8 & 0.5 & 3.0 & 1.9 & 1.2 & 0.9 & 0.6 & 4.4 & 3.3 & 2.2 & 1.7 & 1.1 \\
\hline Isolation & 2.2 & 1.4 & 0.9 & 0.7 & 0.5 & 1.4 & 0.9 & 0.6 & 0.4 & 0.3 & 1.3 & 1.0 & 0.7 & 0.5 & 0.3 \\
\hline General & 3.6 & 2.3 & 1.5 & 1.1 & 0.8 & 0.8 & 0.5 & 0.3 & 0.2 & 0.2 & 1.7 & 1.2 & 0.8 & 0.6 & 0.4 \\
\hline $\begin{array}{l}\text { Antifungal } \\
\text { medication } \\
\text { administration }\end{array}$ & 1.5 & 0.9 & 0.6 & 0.5 & 0.3 & 3.5 & 2.2 & 1.4 & 1.1 & 0.7 & 3.3 & 2.5 & 1.7 & 1.2 & 0.8 \\
\hline $\begin{array}{l}\text { Laboratory } \\
\text { culture }\end{array}$ & 0.1 & 0.1 & 0.1 & 0.0 & 0.0 & 0.2 & 0.1 & 0.1 & 0.0 & 0.0 & 0.2 & 0.1 & 0.1 & 0.1 & 0.0 \\
\hline $\begin{array}{l}\text { Haematologist } \\
\text { consultation }\end{array}$ & 1.6 & 1.0 & 0.7 & 0.5 & 0.3 & 1.3 & 0.8 & 0.5 & 0.4 & 0.3 & 1.2 & 0.9 & 0.6 & 0.4 & 0.3 \\
\hline
\end{tabular}

\section{Discussion}

The patient's age, gender and haematology diagnosis was not statistically significant, with no difference being indicated between the three groups (see Table 4).

The results of the study showed that the overall direct cost of treating an episode of IC was much higher in the group that was treated with fluconazole and an echinocandin, either due to treatment failure or a step down approach, and approximately $85 \%$ more in the echinocandin group when compared to the fluconazole only group. The study by Heimann et al. (2015) supported this finding; their study showed that the mean overall direct treatment costs per patient treated with an echinocandin to be significantly higher than fluconazole, the main contributor being that the echinocandin treated patients were unwell and had longer ICU stays. This is contrary to the findings from Tagliaferri and Menichetti (2015) who found that the echinocandins reduced the overall in-hospital costs compared to fluconazole, and Reboli et al. (2011) who concluded that anidulafungin versus fluconazole in ICU patients resulted in a reduction in total IC related costs due to the decreased length of stay.

Hospital ward costs contributed $76.8 \%$ in the group treated with fluconazole and an echinocandin, $84 \%$ in the fluconazole group and $75.5 \%$ in the echinocandin group, towards the overall direct treatment cost. This outcome is consistent with the findings from the literature review that length of hospital stay has a substantial impact on the cost of treating an episode of IC. Studies using data spanning many years and in various countries conducted by Wilson et al. (2002), Ha et al. (2012), Ceesay et al. (2015) and Armaganidis et al. (2017) all agreed with the findings of this study. From the study results ICU length of stay was much longer (more than double) in the echinocandin group, possibly indicating that this class of antifungal was used in more clinically unwell patients, particularly when compared to the patients treated with fluconazole. This significantly contributed to the overall direct cost where ICU ward fees were ZAR $119692,95 \%$ CI $(43856,195$ 529) in the echinocandin group compared to only ZAR 54 921, 95\% CI (-93 994 - 203 836) in the fluconazole group.

In addition, this study showed that patients in the group treated with both fluconazole and an echinocandin had a much longer mean hospital stay (38.69 days) compared to the fluconazole group (23.3 days) and the echinocandin group (28.45 days). Armaganidis et al. (2017) had a similar result, with patients who switched antifungal treatment having a longer ICU stay, with a mean of 53.8 days.

The second biggest cost contributor, after the hospital wards costs, although much less substantial, were the antifungal treatment costs. The duration of antifungal treatment was the highest in the group treated with both medicines, with a mean of 22.3 days, contributing $16.5 \%$ to the overall direct cost. A similar result was seen in the study by Armaganidis et al. (2017), where patients who switched antifungal therapy had a much longer duration of treatment with a mean of 27.3 days. In this study, the echinocandin group was the second longest, with a mean of 18 days, contributing $17.3 \%$ of the total cost. The fluconazole treatment group was the shortest stay, with a mean of only 8.4 days and a modest $7.5 \%$ toward the overall direct cost. This is reasonably similar to the results of studies 
discussed in the literature review, where Ha et al. (2012) concluded that antifungal treatment costs contributed 10\% of the total direct cost, Heimann et al. (2015) found the treatment costs to be less than $10 \%$ and Wilson et al. (2002) results showed antifungal treatment costs to contribute approximately $17 \%$. Conversely the study conducted by Ananda-Rajah et al. (2011), at an Australian quaternary university-affiliated hospital network, was the only study included in the review that found pharmacy costs to be the main cost contributor, this being attributed to the high acquisition costs of the antifungals that were used as anti-mould prophylaxis and treatment. There was a statistically significant difference in the daily antifungal treatment costs between the groups in this study which was supported by the results of the study by Heimann et al. (2015).

The other two costs factors considered in this study contributed small amounts to the overall treatment cost. The haematologist consultation costs were dependent on the length of hospital stay and the level of care of the patient, as the ICU charge was higher than the general and high care ward consultation costs. The blood culture costs contributed a minor portion towards the overall costs and were independent of the choice of antifungal treatment.

This study had limitations such as a small sample size and only included the private hospital perspective thus the generalizability is questionable.

\section{Conclusion}

The main cost driver in the overall cost of treating IC was due to the ward costs, which contributed $78.8 \%$ on average between the three groups. This was dependent on the level of care of the patients stay, where it can be seen that patients spending longer in the ICU have much higher costs. The antifungal administration costs also contributed a substantial amount to the overall treatment costs, which varied depending on the choice of first line therapy as well as its success, as a change in the treatment resulted in increased treatment costs and extended length of stay. Further studies with larger sample sizes are required to establish whether fluconazole should be used as first line therapy and only changed to an echinocandin where resistance is identified on blood culture.

\section{Ethical Considerations}

The protocol received approval from the Biomedical Research Ethics Committee at the University of KwaZulu-Natal in South Africa (reference number BE403/17). As this was a retrospective chart review study, the patient's permission was not required. However, permission was obtained to access the data from the hospital manager. To prevent any bias, no randomization or blinding in the sample selection was introduced.

\section{Competing Interests Statement}

The authors declare that there are no competing or potential conflicts of interest.

\section{References}

Ananda-Rajah, M. R., Cheng, A., Morrissey, C. O., Spelman, T., Dooley, M., Neville, A. M., \& Slavin, M. (2011). Attributable hospital cost and antifungal treatment of invasive fungal diseases in high-risk hematology patients: an economic modeling approach. Antimicrobial agents and chemotherapy, 55(5), 1953-60. https://doi.org/10.1128/AAC.01423-10

Armaganidis, A., Nanas, S., Antoniadou, E., Mandragos, K., Liakou, K., Koutsoukou, A., ... Zakynthinos, E. (2017). Clinical factors affecting costs in patients receiving systemic antifungal therapy in intensive care units in Greece: Results from the ESTIMATOR study. Mycoses, 60. 454-461. https://doi.org/10.1111/myc.12616

Ceesay, M. M., Sadique, Z., Harris, R., Ehrlich, A., Adams, E. J., \& Pagliuca, A. (April 01, 2015). Prospective evaluation of the cost of diagnosis and treatment of invasive fungal disease in a cohort of adult haematology patients in the UK. Journal of Antimicrobial Chemotherapy, 70(4), 1175-1181. https://doi.org/10.1093/jac/dku506

Cornely, O. A., Aversa, F., Cook, P., Jones, B., Michallet, M., Shea, T., \& Vallejo, C. (January 01, 2011). Evaluating the role of prophylaxis in the management of invasive fungal infections in patients with hematologic malignancy. European Journal of Haematology, 87(4), 289-301. https://doi.org/10.1111/j.1600-0609.2011.01682.x

Gedik, H., Simşek, F., Yıldırmak, T., Kantürk, A., Arıca, D., Aydın, D., Demirel, N., ... Yokuş, O. (January 01, 2014). Primary or secondary antifungal prophylaxis in patients with hematological maligancies: efficacy and damage. Therapeutics and Clinical Risk Management, 10, 305-12. https://doi.org/10.2147/TCRM.S59683

Glöckner, A., \& Karthaus, M. (September 01, 2011). Current aspects of invasive candidiasis and aspergillosis in adult intensive care patients. Mycoses, 54(5), 420-433. https://doi.org/10.1111/j.1439-0507.2010.01885.x

Ha, Y. E., Peck, K. R., Joo, E. J., Kim, S. W., Jung, S. I., Chang, H. H., Park, K. H., ... Han, S. H. (January 01, 2012). 
Impact of first-line antifungal agents on the outcomes and costs of candidemia. Antimicrobial Agents and Chemotherapy, 56(7), 3950-6. https://doi.org/10.1128/AAC.06258-11

Heimann, S. M., Vehreschild, M. J. G. T., von, B.-B. M., Cornely, O. A., Vehreschild, J. J., Vehreschild, M. J. G. T., Vehreschild, J. J., ... Cornely, O. A. (January 01, 2015). Different doses of micafungin for prophylaxis of invasive fungal diseases in hemato-oncological high-risk patients: A web-based non-interventional trial in four large university hospitals in Germany. Transplant Infectious Disease, 16(6), 968-974. https://doi.org/10.1111/tid.12305

Heimann, S. M., Cornely, O. A., Wisplinghoff, H., Kochanek, M., Stippel, D., Padosch, S. A., Langebartels, G., ... \& Vehreschild, J. J. (February 01, 2015). Candidemia in the intensive care unit: analysis of direct treatment costs and clinical outcome in patients treated with echinocandins or fluconazole. European Journal of Clinical Microbiology \& Infectious Diseases, 34, 2, 331-338. https://doi.org/10.1007/s10096-014-2230-8

Kontoyiannis, D. P. (August 01, 2001). A Clinical Perspective for the Management of Invasive Fungal Infections: Focus on IDSA Guidelines. Pharmacotherapy: the Journal of Human Pharmacology and Drug Therapy, 21. https://doi.org/10.1592/phco.21.12.175S.34506

Lancet laboratories 2017, Annual surveillance report \& antibiotic guide. Retrieved 7 May, 2018, from http://www.lancet.co.za/wp-content/uploads/2015/07/Annual-Surveillance-Report-Antibiotic-Guide-SPECI ALIST-PRACTITIONERS-2017.pdf

Playford, E. G., Lipman, J., \& Sorrell, T. C. (October 01, 2010). Prophylaxis, empirical and preemptive treatment of invasive candidiasis. Current Opinion in Critical Care, 16(5), 470-474. https://doi.org/10.1097/MCC.0b013e32833e10e8

Reboli, A. C., Shorr, A. F., Rotstein, C., Pappas, P. G., Kett, D. H., Schlamm, H. T., Reisman, A. L., ... Walsh, T. J. (January 01, 2011). Anidulafungin compared with fluconazole for treatment of candidemia and other forms of invasive candidiasis caused by Candida albicans: a multivariate analysis of factors associated with improved outcome. Bmc Infectious Diseases, 11. https://doi.org/10.1186/1471-2334-11-261

Tagliaferri, E., \& Menichetti, F. (January 01, 2015). Treatment of invasive candidiasis: Between guidelines and daily clinical practice. Expert Review of Anti-Infective Therapy, 13(6), 685-689. https://doi.org/10.1586/14787210.2015.1029916

Wilson, L. S., Reyes, C. M., Stolpman, M., Speckman, J., Allen, K., \& Beney, J. (January 01, 2002). The Direct Cost and Incidence of Systemic Fungal Infections. Value in Health, 5(1), 26-34. https://doi.org/10.1046/j.1524-4733.2002.51108.x

\section{Copyrights}

Copyright for this article is retained by the author(s), with first publication rights granted to the journal.

This is an open-access article distributed under the terms and conditions of the Creative Commons Attribution license (http://creativecommons.org/licenses/by/4.0/). 